\title{
Ureterläsionen bei der Behandlung von Steinen mit der Zeiss-Schlinge: Haftungsrechtliche Problematik*
}

\author{
F. M. Almer, J. E. Altwein \\ Urologische Abteilung des Krankenhauses der Barmherzigen Brüder München
}

\section{Zusammenfassung}

Eine Auswertung der Gerichts-Schlichtungskammern und Versicherungsgutachten 1991 ergab 319 urologische Schadensfälle, an denen Läsionen von Niere und Ureter mit $4(1,3 \%)$ und der Ureter mit 24 $(7,5 \%)$ beteiligt waren. In der eigenen Gutachtensammlung waren seit 1978 bei 4 Fällen eine Ureterverletzung durch eine Zeiss-Schlinge Haftungsgegenstand; dreimal waren eine Nephrektomie, einmal eine End-zu-End-Anastomose notwendig. Die zivilrechtliche Klage wurde einmal zurückgewiesen, einmal in zweiter Instanz abgewiesen, zweimal wurde Schadenersatz (25000 DM bzw. 30000 DM gewährt). Ein Aufklärungsfehler wurde nicht festgestellt. Urologisch wäre je einmal die Nephrektomie bzw. eine Urosepsis bei rechtzeitiger Diagnose und Therapie der Ureterläsion weniger wahrscheinlich gewesen. Gerichtlich wurde ebenfalls genau dies im Sinne einer Schadensbegrenzung bei Komplikationen hervorgehoben.

\section{Ureteral Injuries due to Stone Treatment with the Zeiss Loop: The Problem of Liability}

The evaluation of 319 urological liability files in 1991 yielded 4 renoureteric (1.3\%) and 24 ureteric lesions $(7.5 \%)$. Within 15 years we were required to present an expert opinion in 4 cases of ureteral injury during Zeiss loop stone extraction which resulted in a nephrectomy in 3 patients and an end-to-end anastomosis. All 4 cases went to court. One case was withdrawn before the trial began, in one case the verdict led to the denial of a liability situation in the second trial; in two cases the patient was awarded 25,000 DM and 30,000 DM, respectively. Informed consent was available in all 4 cases, however, the court arrived at the conclusion that the containment of the damage is essential and based upon early recognition and proper management.

\section{Key words}

Ureteral injury - Stone loop - Liability
Die Extraktion von distalen Harnleiterkonkrementen mit der Steinschlinge nach Zeiss ist ein häufiges und wenig risikoreiches Verfahren. Dennoch waren - wie eine Auswertung von Gerichts-, Schlichtungskammernund Versicherungsgutachten von Sparwasser (2) zeigt -, im Zeitraum von 1980 bis 1991 etwa $1 \%$ aller Schadensfälle im urologischen Bereich, die zu einem Haftpflichtverfahren führten, durch diese Behandlungsmethode bedingt. Damit ist diese Methode - abgesehen von Verletzungen bei gynäkologischen Operationen - die häufigste Ursache von Haftpflichtverfahren hinsichtlich iatrogener Ureterschäden mit schweren Komplikationen bis hin zur Nephrektomie.

Bei der Auswertung von vier Gutachten aus unserer eigenen Sammlung zu diesem Thema fiel eine große „Entscheidungsbreite" hinsichtlich des Ausganges
Tab. 1 Kasuistik

\begin{tabular}{|c|c|c|c|}
\hline & Diagnose & med. Ausgang & jur. Ausgang \\
\hline Fall 1 & $\begin{array}{l}\text { prävesikaler } \\
\text { Ureterstein rechts }\end{array}$ & $\begin{array}{l}\text { Sepsis, akutes } \\
\text { Abdomen, verzögerte } \\
\text { Nephrektomie }\end{array}$ & $\begin{array}{l}25000 D M \\
\text { Schadens- } \\
\text { ersatz }\end{array}$ \\
\hline Fall 2 & $\begin{array}{l}\text { V.a. hoher Ureter- } \\
\text { stein links }\end{array}$ & $\begin{array}{l}\text { starke Flanken- } \\
\text { schmerzen, sofort } \\
\text { Nierenfreilegung, } \\
\text { Nephrektomie }\end{array}$ & $\begin{array}{l}\text { Klage zurück- } \\
\text { gezogen }\end{array}$ \\
\hline Fall 3 & $\begin{array}{l}\text { hoher Ureterstein } \\
\text { rechts }\end{array}$ & $\begin{array}{l}\text { Sepsis, Schock, } \\
\text { verzögerte offene } \\
\text { Fistelung, End-zu- } \\
\text { End-Anastomose }\end{array}$ & $\begin{array}{l}30000 \mathrm{DM} \\
\text { Schadens- } \\
\text { ersatz }\end{array}$ \\
\hline Fall 4 & $\begin{array}{l}\text { prävesikaler } \\
\text { Ureterstein links }\end{array}$ & $\begin{array}{l}\text { Fieber, Schmerzen, } \\
\text { verzögerte } \\
\text { Nephrektomie }\end{array}$ & $\begin{array}{l}\text { Klage in } 2 \text {. } \\
\text { Instanz abge- } \\
\text { wiesen }\end{array}$ \\
\hline
\end{tabular}


der eingeleiteten Haftpflichtverfahren auf (Tab. 1). Diese Fälle sollen im folgenden diskutiert werden.

\section{Sachverhalt}

\section{Fall: LG Traunstein AZ 50 282/78}

Stationäre Aufnahme eines 46jährigen Patienten nach rechtsseitigen Nierenkoliken. Im IUG zeigte sich ein kleiner prävesikaler Harnleiterstein rechts. Es folgte die Anlage einer Zeiss-Schlinge in ITN, die auf eine Höhe von $10 \mathrm{~cm}$ herabgezogen wurde. Nach drei Tagen Schlingenextraktion. Es entwickelten sich septische Fieberschübe und ein paraureteres Kontrastmittelextravasat. Therapie: hochdosierte intravenöse Antibiose. Entlassung nach drei Wochen bei Beschwerdefreiheit des Patienten. Nach weiteren zwei Wochen kam es zur Wiederaufnahme des nun hochfieberhaften Patienten mit akutem Abdomen. Das IUG zeigte nun ein großes Kontrastmittelextravasat im Bereich des mittleren Harnleiterdrittels rechts sowie eine Stauung III-IV nach Emmett. Man entschloß sich zur Freilegung der rechten Niere. Intraoperativ imponierte eine vollständige Verschwartung des gesamten Retroperitoneums. Der abgerissene Harnleiter konnte nicht freigelegt werden; der Patient wurde nephrektomiert.

\section{Fall: LG Saarbrücken AZ $160300 / 85$}

Stationäre Aufnahme einer 49jährigen Patientin wegen rezidivierender Koliken links. Die Übersichtsaufnahme ergab keinen sicheren Anhalt für ein Konkrement. Bei Fortdauer der Beschwerden Entschluß zur Anlage einer Zeiss-Schlinge links, die langsam bis zu einem Widerstand heruntergezogen wurde. In der darauffolgenden Nacht klagte die Patientin über starke linksseitige Flankenschmerzen. Ob die Patientin mit der Schlinge hängengeblieben war, konnte nicht geklärt werden. Am nächsten Tag daraufhin Schlingenextraktion, wobei sich ein umgestülpter Harnlejter zeigte. Am selben Tag linksseitige Nierenfreilegung. Hierbei fand sich ein kompletter Abriß des Nierenbeckens an der linken Niere. Es wurde die Nephrektomie links angeschlossen.

\section{Fall: LG Landshut AZ O 903/87}

Stationäre Aufnahme einer 56jährigen Patientin wegen kolikartiger Flankenschmerzen rechts. Nach zunächst konservativer spasmolytischer Therapie zeigte das retrograde Pyelogramm rechts einen linsengroßen Stein im Bereich des Überganges vom proximalen zum mittleren Harnleiterdrittel. Der Versuch, eine Zeiss-Schlinge an diesem Stein vorbeizuführen, scheiterte in Höhe des Steins. Der endoskopische Eingriff wurde damit abgebrochen. Noch am selben Tag entwickelten sich Anzeichen eines septischen Schocks. Es wurde mit kreislaufunterstützenden Medikamenten und parenteraler Antibiotikagabe reagiert. Ein erneutes retrogrades Pyelogramm einen Tag später zeigte einen Kontrastmittelübertritt aus dem Harnleiterlumen in Höhe des Steinbettes in den Retroperitonealraum. Die daraufhin versuchte Harnleiterschienung mißlang, die Spitze der Harnleiterschiene verließ wiederholt in Höhe des Steinbettes den Harnleiter. Nach weiteren 2 Tagen erfolgte die Verlegung der Patientin in eine andere Klinik, wo sofort die lumbale Nierenfreilegung, die offene Nierenfistelung, die Resektion des durchblutungsgestörten Harnleitersegmentes im Bereich der Perforationsstelle und End-zu-End-Anastomose durchgeführt wurden.

\section{Fall: OLG Hamm AZ 3 U 124/87}

Stationäre Aufnahme eines 51jährigen Patienten wegen Harnleiterkoliken links. Diagnose eines prävesikalen Konkrementes links. Da der Patient auf schnelle Behandlung drängte, wurde bereits am nächsten Tag eine Entfernung des Harnleitersteines mittels der Zeiss'schen Schlinge in Narkose durchgeführt. Entlassung aus der stationären Behandlung einen Tag später. Nach einem weiteren Tag begab sich der Patient wieder in ambu- lante Behandlung wegen Unwohlseins und erhöhten Temperaturen. Das IUG zeigte ein großes Paravasat links, das Urinsediment war ohne pathologischen Befund. Es folgte die ambulante $\mathrm{Be}-$ handlung mit einem Breitbandantibiotikum. Trotz starker Schmerzen und Beschwerden stellte sich der Patient erst wieder nach einer Woche ambulant vor. Ein erneutes IUG zeigte nun ein ausgedehntes Paravasat im Retroperiteonealraum unterhalb der linken Niere. Bei der am folgenden Tag durchgeführten Nierenfreilegung war eine End-zu-End-Anastomose nicht mehr möglich, man entschied sich zur Nephroureterektomie links.

\section{Gutachterliche Beurteilung}

\section{Fall}

Die Ureterläsion an und für sich läßt nicht den Rückschluß auf eine Verletzung der Sorgfaltspflicht im Sinne eines sog. Kunstfehlers zu. Jedoch hätte danach aufgrund der Zeichen einer infizierten Harnstauungsniere mit Kontrastmittelextravasation operativ eingegriffen werden müssen, beispielsweise Nephrostomie rechts und ausgiebige Drainage des Retroperitonealraumes. Eine operative Behandlung hätte im Gegensatz zur lediglich konservativ erfolgten Therapie die rechte Niere mit hoher Wahrscheinlichkeit gerettet.

\section{Fall}

Die Anlage einer hohen Harnleiterschlinge links aufgrund der weiterhin bestehenden Beschwerden der Patientin und des Verdachtes auf ein Harnsäurekonkrement wird als indiziert bewertet. Das Legen und Entfernen war mit der erforderlichen Sorgfalt erfolgt, eine radiologische Kontrolle hatte aber nicht stattgefunden, was jedoch nicht zwingend erforderlich war. Der Zeitpunkt des Harnleiterabrisses konnte nicht eindeutig festgestellt werden. Ein Harnleiterabriß am Nierenbecken bei Schlingenextraktion, wie es der intraoperative Befund zeigte, erfolgt in der Regel laut Gutachten, wenn die Schlinge bei der Extraktion oberhalb der Gefäßkreuzung plaziert ist und gegen einen Widerstand gezogen wird. Ob der Harnleiterabriß während der Schlingenextraktion zustande kam oder dadurch, daß die Patientin mit der Schlinge hängengeblieben war, konnte im Nachhinein nicht mehr eindeutig festgestellt werden. Auch eine lege artis durchgeführte Extraktion einer hohen Verweilschlinge bringt immer das Risiko eines Harnleiterabrisses mit sich. Die Nephrektomie war geboten, weil eine plastische Operation aufgrund des nach distal gezogenen Harnleiters nicht mehr durchzuführen war. Auch eine Fistelung der linken Niere als Dauerlösung hätte die Lebensqualität der Patientin stark beeinträchtigt. Die Nephrektomie war hier Therapie der Wahl.

\section{Fall}

Bei fehlgeschlagenem Zeiss-Versuch wurde versäumt, sofort eine potentiell mögliche Harnleiterverletzung durch Kontrastmittelfüllung des Harnleiters nachzuweisen. Spätestens nach dem am nächsten Tag erfolgten retrograden Kontrollpyelogramm hätte eine sofortige Freilegung und Drainage des Retroperitonealraumes erfolgen müssen. Diese Maßnahme hätte die Ausbildung eines septischen, lebensbedrohlichen Krankheitsbildes mit hoher Wahrscheinlichkeit vermeiden können. In der Klageschrift war (im Gegensatz zu Fall 2) der Vorwurf erhoben worden, daß die Steinextraktion mit der Steinschlinge im Vergleich mit anderen Therapiemethoden im Falle eines hohen Uretersteines der Eingriff mit dem höheren Risiko sei. $\mathrm{Zu}$ den Alternativmethoden wurde festgestellt, daß die Ultraschallzertrümmerung von Harnleitersteinen im Rahmen der Ureterorenoskopie eindeutig das Verfahren mit der höheren Invasivität sei. Die extrakorporale Stoßwellenlithotripsie hingegen stellte 1986 bei der Behandlung von hohen Harnleitersteinen eine anerkannte, mit hohem Prozentsatz erfolgreiche Therapieform dar. Die ESWL war im Vergleich zur Zeiss-Schlinge das Verfahren mit der geringeren Invasivität. 


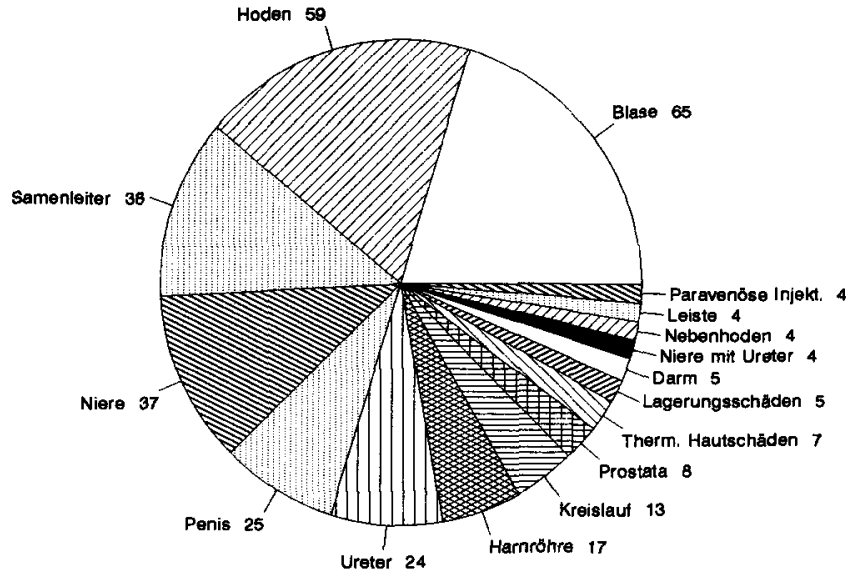

Abb. 1 Schadensfälle nach Organbezug $(n=319)$ (nach Sparwasser 1991)

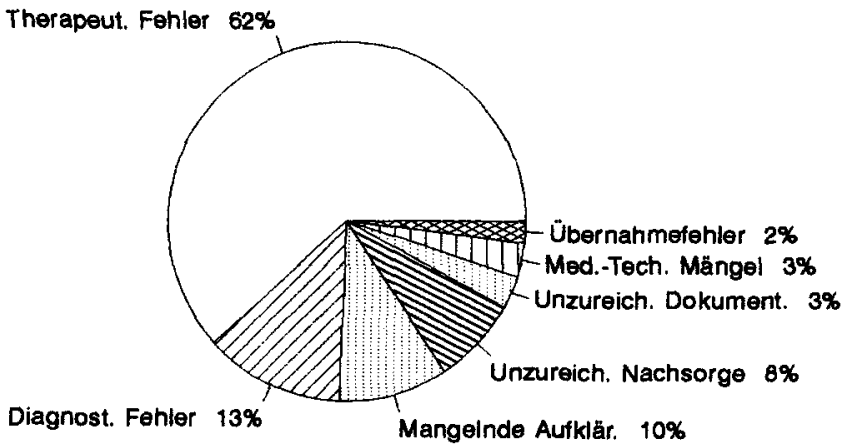

Abb. 2 Analyse der Schadenskategorien (nach Spanwasser 1991)

\section{Fall}

Die Schlingenextraktion war indiziert. Auch bei sachgemäßer Durchführung besteht immer ein minimales Risiko der Harnleiterverletzung. Eine radiologische Kontrolle bei der Extraktion war nicht unbedingt notwendig. Das Vorliegen eines Paravasates nach dem Eingriff war für sich gesehen noch kein Grund für einen operativen Eingriff, es mußte erst ein akutes Krankheitsbild dazukommen, um das Operationsrisiko zu rechtfertigen. Eine Mehrheit der Fachärzte hätte allerdings schon in diesem Stadium eingegriffen.

\section{Erledigung der Verfahren}

\section{Fall}

Im Urteil wurde dem Kläger Recht gegeben. Ihm wurde eine Summe von 25000 DM als Schadensersatz zuerkannt.

\section{Fall}

Nach Eingang des Gutachtens zog die Klägerin ihren Antrag auf Schadensersatz zurück.

\section{Fall}

Die Klägerin bekam Recht. Ihr wurde Schadensersatz von 30000 DM zuerkannt.

\section{Fall}

In dem Urteil des Gerichtes wird offengelassen, ob der Kläger über das Risiko einer Verletzung des Harnleiters hätte aufgeklärt werden müssen, was nicht geschehen war. Der Kläger hätte sich auch frühzeitig wieder ambulant vorstellen müssen, nachdem stärkere Beschwerden aufgetreten waren. Die Klage wurde in zweiter Instanz abgewiesen.

\section{Diskussion}

Sparwasser (2) hat mit einer bundesweiten Umfrage bei Haftpflichtversicherern herausgefunden, daß bei 24 von 319 Schadensfällen im urologischen Bereich eine Ureterverletzung und bei 4 Fällen eine Ureterverletzung mit nachfolgendem Nierenschaden vorlag (Abb. 1). Diese Verletzungen waren hauptsächlich die Folge von gynäkologischen Operationen. Der Anteil von Ureterläsionen durch die Zeiss-Schlinge betrug etwa $1 \%$. Die Analyse der Schadenskategorien, d. h. vorliegenden Behandlungsfehler ergab als Hauptschadensursache den therapeutischen Fehler, gefolgt von Diagnosefehlern. Erst an dritter Stelle stand die mangelnde Aufklärung ( $\mathrm{Abb} .2$ ). Bei den beteiligten Beilegungsinstanzen zeigt sich von 1980 bis 1989 ein Trend weg vom Gericht und hin zur Schlichtungsstelle (Abb. 3), wobei die Entscheidungshäufigkeit zugunsten des Arztes eindeutig bei den Schlichtungsstellen am größten ist (Abb. 4).

Nach wie vor ist die Steinextraktion mit der Zeiss-Schlinge ein häufig angewendetes Verfahren in der Urologie. Vor dem Einsatz der Zeiss'schen Schlinge sollte nach Vahlensieck (4) in der Regel ein konservativer Therapieversuch stehen, da hier mit 80-90\% Erfolgsrate zu rechnen sei. Eine sofortige manuelle Extraktion komme nur bei tiefsitzenden Harnleitersteinen in Frage.

Bereits 1951 dokumentieren Ellik u. Mitarb. (4) insgesamt 4 Ureterperforationen $(3,8 \%)$ in einer Serie von 104 durchgeführten Ureterkonkrement-Extraktionen mit der Steinschlinge. Bei 3 der Patienten lag ein hohes Ureterkonkrement vor. Bei allen 4 Patienten erfolgte die Ureterschienung und retroperitoneale Drainage, in einem Fall mußte die Nephrektomie angeschlossen werden.

In einer Serie von 147 endokopischen Eingriffen bei mittleren und distalen Ureterkonkrementen berichten Weinberg u. Mitarb. (5) über insgesamt 15 akute Komplikationen, d. h. in 10 Prozent aller Eingriffe. Es handelte sich dabei um einen Ureterabriß $(0,7 \%)$, zwei Ureterperforationen (1\%), 11 Fälle von postoperativem Fieber ( $7 \%$ ) und einen Fall von transfusionspflichtigem Blutverlust $(0,7 \%)$.

Die Schlingenextraktion birgt somit bei hoher Erfolgsrate das geringe Risiko einer schweren Harnleiterverletzung, wobei das Risiko abhängig von der Lokalisation des Konkrementes ist. In allen vier dargestellten Schadensersatz-Fällen war es zu Harnleiterabriß oder Perforation mit nachfolgenden starken Schmerzen, septischen Temperaturen und schließlich operativer Revision gekommen. Die Anlage und Extraktion einer Zeiss-Schlinge wurde von gutachterlicher Seite bei allen vier Patienten als in- 


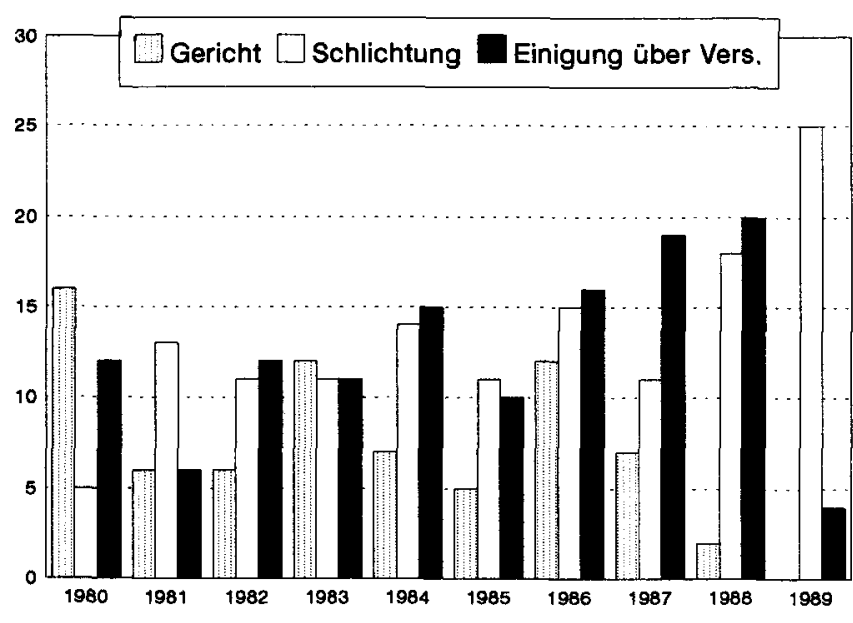

Abb. 3 Beilegungsinstanzen 1980-1989 (nach Sparwasser 1991)

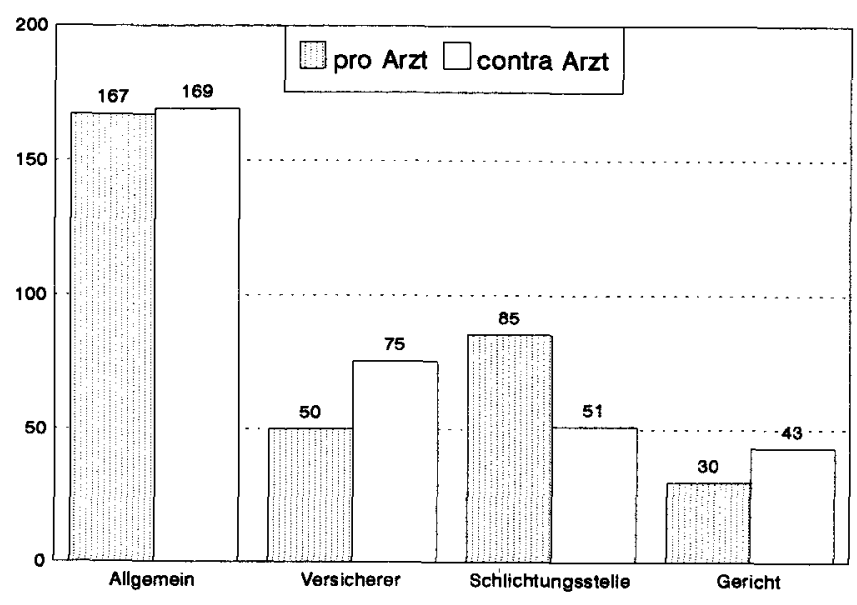

Abb. 4 Entscheidungshäufigkeit nach Beilegungsinstanzen pro \& contra Arzt $(n=339)(n=319)$ (nach Sparwasser 1991)

diziert und fachgerecht ausgeführt bewertet. Eine Durchleuchtungskontrolle wurde als wünschenswert, aber nicht unbedingt notwendig erachtet. Auf die Lokalisation des Harnleitersteines als Grundlage der Indikation ist nur in einem Fall Bezug genommen worden: bei Fall Nr. 3 wurde auf die risikoärmere Alternativmethode ESWL bei hohen Harnleitersteinen hingewiesen.

Ulsenheimers (3) Klassifikation der Behandlungsfehler, die aus den häufigsten Fehlleistungen typische Fallgruppen bildet, macht einen systematischen Überblick über die Gründe möglich, die zu der großen juristischen Entscheidungsbreite bei medizinisch ähnlichem Ausgang führten. Insbesondere sind hier zu diskutieren:

Diagnosefehler, falsche Indikationsstellung: In keinem der Fälle wurde die Diagnose eines Ureterkonkrementes oder die Indikation zur Anlage einer Zeiss-Schlinge angezweifelt, auch nicht in Fall 2 bei nur klinischem Verdacht auf ein Uratkonkrement. Zudem wurden in den Fällen 1, 2 und 4 die Nephrektomie als indiziert gewertet.
Falsche Wahl der Heilmethode:

In Fall 3 (hoher Ureterstein) wurde festgestellt, da $\beta$ unter mehreren anerkannten Heilmethoden nicht diejenige ausgewählt worden war, die die geringste Gefahr für den Patienten bedeutet (ESWL). Dies führt unter anderem zur Verurteilung.

Fehlerhafte Operationstechnik:

In keinem der Fälle wurde der endourologische Eingriff als nicht fachgerecht ausgeführt gewertet. Auch die fachgerechte Anlage und Extraktion der Steinschlinge birgt immer das Risiko von Ureterverletzungen in sich.

Nichterkennen von Komplikationen:

Trotz Kontrastmittelextravasation, Schmerzen und septischen Fieberschüben wurde die operative Intervention in Fall 1 und 3 nicht frühzeitig genug durchgeführt. Hätte man - wie in Fall 2 - adäquat reagiert, so wäre es wahrscheinlich weder zum Nierenverlust im 1 . Fall, noch zu einer lebensbedrohlichen Urosepsis im 3. Fall gekommen. Im 4. Fall hielt der Gutachter bei zunächst kleinem Paravasat und normalem Urinstatus eine abwartende Haltung für vertretbar, wobei seiner Meinung nach die Mehrheit der Urologen eine sofortige Operation befürwortet hätten. Die verspätete Operation mit resultierendem Verlust der Niere wurde durch den Patienten selbst verschuldet, der sich bei bestehenden starken Schmerzen und Fieber nicht frühzeitig genug dem behandelnden Urologen wieder vorstellte.

Erwähnenswert ist, daß auf eine mangelnde Risikoaufklärung nur im 4. Fall eingegangen wurde. In welchem Umfang eine Aufklärung hätte erfolgen müssen, ließ das Gericht offen. Es war jedoch der Überzeugung, daß der Patient, der auf eine schnelle Behandlung drängte, das minimale Risiko einer folgenreichen Harnleiterverletzung auf sich genommen hätte.

Da jedoch zunehmend häufig der Vorwurf unzureichender oder fehlender Aufklärung erhoben wird, sollte präoperativ in jedem Falle der Hinweis auf notwendig werdende Operationen nach möglichen Ureterverletzungen gegeben und schriftlich festgehalten werden. Es sei aber hier noch einmal darauf hingewiesen, da $B$ in den besprochenen Fällen eine eingehende Aufklärung am Ausgang der gerichtlichen Verfahren nichts geändert hätte!

\section{Schlußfolgerung}

Gerichtlich wurde festgestellt, daß nach Eintritt eines Schadens durch Steinschlingen-Extraktion (Ureterperforation oder -abriß) mit radiologisch nachgewiesenem Kontrastmittelextravasat dieser Schaden durch eine indizierte sofortige Operation zu begrenzen ist und nicht auch noch zum Nierenverlust führen muß.

Hinzu kommt noch die ärztliche Aufklärungsproblematik, die jedoch bei den 4 besprochenen Fällen nicht im Vordergrund steht. In allen Fällen handelte es sich um Schadensersatzansprüche, wobei das Nichterkennen der Komplikationen auch strafrechtliche Folgen hätte haben können. 


\section{Anmerkung}

Wir danken Herrn Prof. Dr. W. Eisenmenger, Vorstand des Instituts für Rechtsmedizin der Universität München, für die freundliche juristische Beratung.

\section{Literatur}

1 Ellik, M., L. A. Newton: Ureteral calculi: Experiences in looped catheter management. J. Urol. 65 (1951) 532-536

2 Sparwasser, H.H.: Übersicht über Auswertung von Gerichts-, Schlichtungskammern- und Versicherungsgutachten, aufgegliedert in typische Risiken. Vortrag 32. Tagg. Südwestdeutsche Gesellschaft für Urologie 9. -11. 4. 1991

3 Ulsenheimer, $K$.: Arztstrafrecht in der Praxis, Verlag Decker u. Müller, Heidelberg (1988)

4 Vahlensieck, W.: Das urologische Haftpflichtgutachten. In: Baumbusch, F., E. Schindler, Th. Schultheis, W. Vahlensieck: Die urologische Begutachtung und Dokumentation. Handbuch der Urologie VII/2, Springer Verlag, Berlin (1965) 474-475

5 Weinberg, J. J., A.D. Smith: Complications of ureteroscopy, in: Smith, R. B., R. M. Ehrlich: Complications of urologic surgery, Saunders, Philadephia (1990) 277-285

\section{Frank M. Almer}

Urologische Abteilung

Krankenhaus der Barmherzigen Brüder

Romanstr. 93

80639 München 